\title{
Effects of dietary fat quantity and composition on fasting and postprandial levels of coagulation factor VII and serum choline-containing phospholipids
}

\author{
Anja Schou Lindman ${ }^{1}$, Hanne Müller ${ }^{1}$, Ingebjørg Seljeflot ${ }^{2}$, Hans Prydz ${ }^{3}$, Marit Veierød ${ }^{4,5}$ \\ and Jan I. Pedersen ${ }^{1,5 *}$ \\ ${ }^{1}$ University College of Akershus, 1356 Bekkestua, Norway \\ ${ }^{2}$ Center for Clinical Research, Ullevål University Hospital, 0407 Oslo, Norway \\ ${ }^{3}$ Biotechnology Centre of Oslo, University of Oslo, 0317 Oslo, Norway \\ ${ }^{4}$ Section of Medical Statistics, University of Oslo, 0316 Oslo, Norway \\ ${ }^{5}$ Institute for Nutrition Research, University of Oslo, 0316 Oslo, Norway
}

(Received 4 November 2002 - Revised 27 March 2003 - Accepted 12 April 2003)

\begin{abstract}
Dietary fat influences plasma levels of coagulation factor VII (FVII) and serum phospholipids (PL). It is, however, unknown if the fatmediated changes in FVII are linked to PL. The present study aimed to investigate the effects of dietary fat on fasting and postprandial levels of activated FVII (FVIIa), FVII coagulant activity (FVIIc), FVII protein (FVIIag) and choline-containing PL (PC). In a randomized single-blinded crossover-designed study a high-fat diet (HSAFA), a low-fat diet (LSAFA), both rich in saturated fatty acids, and a high-fat diet rich in unsaturated fatty acids (HUFA) were consumed for 3 weeks. Twenty-five healthy females, in which postprandial responses were studied in a subset of twelve, were included. The HSAFA diet resulted in higher levels of fasting FVIIa and PC compared with the LSAFA and the HUFA diets (all comparisons $P \leq 0 \cdot 01$ ). The fasting PC levels after the LSAFA diet were also higher than after the HUFA diet $(P<0 \cdot 001)$. Postprandial levels of FVIIa and PC were highest on the HSAFA diet and different from LSAFA and HUFA (all comparisons $P \leq 0 \cdot 05)$. Postprandial FVIIa was higher on the HUFA compared with the LSAFA diet $(P<0 \cdot 03)$, whereas the HUFA diet resulted in lower postprandial levels of PC than the LSAFA diet $(P<0.001)$. Significant correlations between fasting levels of PC and FVIIc were found on all diets, whereas FVIIag was correlated to PC on the HSAFA and HUFA diet. The present results indicate that dietary fat, both quality and quantity, influences fasting and postprandial levels of FVIIa and PC. Although significant associations between fasting FVII and PC levels were found, our results do not support the assumption that postprandial FVII activation is linked to serum PC.
\end{abstract}

Coagulation factor VII: Phospholipids: Dietary fat

Several clinical studies have reported an association between increased levels of coagulation factor VII (FVII) and the risk of cardiovascular disease (Dalaker et al. 1985; Meade et al. 1986; Heinrich et al. 1994; Miller et al. 1996), although diverging results exist (Folsom et al. 1993; Junker et al. 1997; Cooper et al. 2000). FVII is an essential enzyme involved in the extrinsic pathway of blood coagulation. The major proportion of FVII circulates in the zymogen form as a single-chain glycoprotein. Low, but significant levels (about $1 \%$ ) of activated factor VII (FVIIa) are also present in plasma and appear to serve as a primer for triggering the clotting cascade. In earlier clinical studies, demonstrating significant associations between cardiovascular disease and FVII, the two most common laboratory methods used for FVII measurements reflected the total amount of FVII protein (FVIIag) or FVII coagulant activity (FVIIc). A new method for determining FVIIa was commercialized in the mid-1990s (Morrissey et al. 1993). Until now only one cross-sectional study using this method has shown association between FVII and cardiovascular disease (Miller et al. 1996), whereas most others have failed to demonstrate this association (Merlini et al. 1995; Moor et al. 1995; Cooper et al. 2000; Eriksson-Berg et al. 2001). These findings support the assumption that the rise in FVII levels in patients with cardiovascular disease observed in the early studies might be partially explained by a rise in FVIIag concentration rather than by an increase in FVIIa. The clinical

Abbreviations: FVII, coagulation factor VII; FVIIa, activated FVII; FVIIag, FVII protein; FVIIc, FVII coagulant activity; HSAFA, high-fat diet rich in saturated fatty acids; HUFA, high-fat diet rich in unsaturated fatty acids; LSAFA, low-fat diet rich in saturated fatty acids; PC, choline-containing phospholipids; PL, phospholipids.

* Corresponding author: Professor Jan I. Pedersen, fax +47 228513 41, email j.i.pedersen@basalmed.uio.no 
impacts of elevated FVII levels are still controversial and it still remains unclear whether or not any forms of FVII are related to the risk of cardiovascular disease.

It is now fairly well established that FVIIa and FVIIc are influenced by dietary fat quantity, and to some extent also fat composition, whereas FVIIag is unaffected by dietary fat (Mennen et al. 1996; Larsen et al. 1999, 2000; Sanders et al. 2000, 2001). In addition to the increased levels of fasting FVIIc after high-fat diets compared with low-fat diets observed in several studies (Miller et al. 1986; Marckmann et al. 1993; Brace et al. 1994), increased postprandial levels of FVIIa and FVIIc have been demonstrated (Kapur et al. 1996; Mennen et al. 1998; Oakley et al. 1998; Sanders et al. 2000). The mechanisms responsible for these observed fat-mediated changes in plasma FVII levels are unknown.

An association between blood lipids and FVII has been investigated by several authors (Marckmann et al. 1990, 1992, 1993; Kapur et al. 1996; Larsen et al. 2000; Sanders et al. 2000, 2001), but no conclusion has been reached. However, an influence of serum phospholipids (PL) on FVII has been proposed. In the study of Dalaker et al. (1985), they found increased levels of FVIIc in men at high risk for cardiovascular disease and demonstrated that this was due to a PL-FVII complex in plasma. Furthermore, Mariani et al. (1999) reported in a recent study that serum choline-containing PL (PC) were the main determinants of FVIIa and FVIIc in two common FVII genotypes. The influence of dietary fat on serum PL concentration has not been extensively studied, but Havel (1957) reported that serum PL increased significantly after a fat-rich meal.

The aim of the present study was to compare the effects of a high-fat diet rich in saturated fatty acids (HSAFA), a low-fat diet rich in saturated fatty acids (LSAFA) and a high-fat diet rich in unsaturated fatty acids (HUFA) on fasting and postprandial levels of FVII, and PC and their possible associations. The present study was part of a larger study aiming to investigate the effects of fatty acids on blood lipids (Muller et al. 2003).

\section{Methods}

\section{Subjects}

The thirty-one healthy female subjects initially enrolled were all students in home economics, recruited from the University College of Akershus, Norway. There were no screening criteria with regard to smoking habits, age or physical activity. The exclusion criteria were chronic use of medications, obesity (BMI $>32 \mathrm{~kg} / \mathrm{m}^{2}$ ) and pregnancy. Seven subjects were on oral contraceptives and one had a hormone-releasing intra-uterine device. Nine subjects were smokers. The mean age of the participants was 30.5 (SD 9.8) years, weight 67.4 (SD 12.1) kg, and BMI 24.5 (SD 3.2$) \mathrm{kg} / \mathrm{m}^{2}$. The study protocol was carefully explained to the participants before they entered the study and they all gave their written consent. The subjects received free food, but no payment during the study. The trial was approved by the Regional Committee for Ethics in Biomedical Research.

\section{Study design}

In the single-blinded, strictly controlled, dietary crossover study, the participants were randomly allocated to consume the HSAFA diet (A), the LSAFA diet (B) and the HUFA diet $(C)$ for periods of 3 weeks in one of three sequences $(\mathrm{ABC}, \mathrm{BCA}, \mathrm{CAB})$. The intervention periods were separated by a 1-week washout period, in which the subjects returned to their normal eating habits. Fasting responses to the diets were studied in all thirty-one participants, whereas postprandial responses were studied in a subset of thirteen (voluntary participation).

The participants were requested to maintain their normal lifestyle and level of physical activity throughout the study, and informed to abstain from alcohol consumption. They were only allowed to eat and drink the experimental diets, except for coffee, tea and mineral water with an artificial sweetener.

\section{Anthropometric measurements}

Body weight in the fasting state was measured at study entry, and after each intervention period, and also in the non-fasting state before dinner twice weekly. A digital balance weight was used, read to the nearest $0.1 \mathrm{~kg}$. Heights were registered at inclusion, and BMI calculated as weight $(\mathrm{kg}) /$ height $(\mathrm{m})^{2}$.

\section{Experimental margarines}

Two different test margarines were used. One margarine was rich in saturated fatty acids and based on $(\mathrm{g} / \mathrm{kg})$ : coconut oil, 800; soyabean oil, 100; rapeseed oil, 100 (saturated fatty acid margarine). The other was a commercially available margarine rich in unsaturated fatty acids that consisted of palm oil, refined sunflower-seed oil, coconut oil and refined rapeseed oil (unsaturated fatty acid margarine). Due to its content of sunflower-seed oil, the unsaturated fatty acid margarine contained more tocopherol than the saturated fatty acid margarine $(35.5$ and $16.4 \mathrm{mg} / 100 \mathrm{~g}$, respectively). Both margarines contained $16 \%$ water.

\section{Experimental diets}

Three diets were designed by using a computer-based, nutrient-calculation program ("Mat på data" 3.0c; the National Association for Nutrition and Health, Oslo, Norway). Two diets were based on the saturated fatty acid margarine and were planned to contain 42 and $22 \%$ energy as fat (HSAFA and LSAFA, respectively). The third diet was based on the unsaturated fatty acid margarine and was planned to contain $42 \%$ energy as fat (HUFA). The diets were all based on a $7 \mathrm{~d}$ menu and designed to be isoenergetic. The HSAFA and HUFA diets were identical except for the test fat. The removed fat in the LSAFA diet was replaced by food items rich in carbohydrates; fruits, orange juice and sweets. The fat from the background diet was calculated to supply $7.8 \%$ energy in all diets, and came from meat, fish, cereals and dairy products. The test margarines were incorporated into bread, cakes, 
buns, porridge and sauces for dinner, in addition to be used as spread.

All meals consumed were precisely weighed for each individual. Breakfast, lunch, evening meals and snacks were packed and ready for home consumption. Dinner was prepared at the college and served under supervision in a dining room every day except during the weekend. Weekend meals were prepared and packed for home consumption. All perishable foods were provided frozen. For snacks all diets contained cake, yoghurt, fruit, juice and, for the LSAFA diet, sweets.

Body weight, height and level of physical activity were used for assessment of individual energy intake (World Health Organization, 1985). The subjects were supplied with food to meet $100 \%$ of their mean daily energy requirement. If the participants temporarily increased their activity or lost weight, they were allowed to eat buns with the same fat composition as the rest of the diet or their energy intake was adjusted.

\section{Chemical analysis}

Duplicate portions corresponding to a daily energy intake of $8.2 \mathrm{MJ}$ were collected of the three diets. The duplicate portions were kept frozen at $-20^{\circ} \mathrm{C}$. After homogenization and freeze-drying, the homogenates from 7 weekdays were pooled into one portion for each diet, and fatty acid composition, energy, $\mathrm{N}$, fat and cholesterol content were analysed as previously described (Muller et al. 2001).

\section{Blood sampling procedures and laboratory methods}

Fasting blood samples of all subjects were collected after an overnight fast at the study entry and on the last day in each period. On the same day, postprandial samples of the subset of participants were collected at 09.30, 12.30, 16.00 and 20.30 hours, $1.5 \mathrm{~h}$ after each meal. In addition, a second fasting sample from the subset was collected the next day.

Both fasting and postprandial blood samples were collected by venipuncture using minimal stasis. Blood for analyses of FVII (FVIIa, FVIIc, FVIIag) were collected in $5 \mathrm{ml}$ vacutainer tubes (Becton Dickinson, Plymouth, Devon, UK) containing $0.5 \mathrm{ml}$ sodium citrate $(0.129 \mathrm{M})$ and separated within $45 \mathrm{~min}$ by centrifugation at $2500 \mathrm{rpm}$ for $30 \mathrm{~min}$ at room temperature (to avoid cold-activation). Blood for PC analysis was collected in tubes without anticoagulant, and serum was separated within $60 \mathrm{~min}$ at $2500 \mathrm{rpm}$ for $15 \mathrm{~min}$ at $4^{\circ} \mathrm{C}$.

FVIIa, FVIIc and PC of both fasting and postprandial samples were analysed, whereas FVIIag was only measured in samples collected in the fasting state. All samples from each subject were analysed in duplicate and in a single run. Plasma samples for coagulation analyses were rapidly thawed in a water bath at $37^{\circ} \mathrm{C}$ and analysed within $1.5 \mathrm{~h}$. FVIIa was determined by a one-step clotting assay (Staclot VII-rTF; Diagnostica Stago, Asniere, France). The standard was a recombinant FVIIa supplied with the kit. Values are expressed in $\mathrm{mU} / \mathrm{ml}$, $30 \mathrm{mU}$ being equivalent to $1 \mathrm{ng}$ FVIIa. FVIIag was determined by ELISA (Asserachrom VIIag; Diagnostica
Stago). Values are expressed as ng/ml. FVIIc was analysed by using a two-step chromogenic assay containing human placenta thromboplastin (Coa-Set FVII; Chromogeni AB, Mölndal, Sweden), and are presented as percentage of a standard. Serum samples for PC determination were thawed on ice. PC was measured by an enzymic colorimetric test according to Takayama et al. (1977) (Test Combination Phospholipids; Boehringer Mannheim, Mannheim, Germany). Values are expressed as $\mathrm{mmol} / \mathrm{l}$. The interassay CV were $3.1 \%$ for FVIIc, $6.2 \%$ for FVIIag and $6.2 \%$ for PC. For FVIIa the interassay CV for the controls supplied with the kit were $3.3 \%$ for the low $(60 \mathrm{mU} / \mathrm{ml})$ and $12.4 \%$ for the high $(165 \mathrm{mU} / \mathrm{ml})$ concentration control. FVIIa results from participants having levels $>200 \mathrm{mU} / \mu \mathrm{l}$ in at least one sample were excluded because of analytical imprecision above this level.

\section{Dietary compliance}

Dietary compliance during the intervention periods was judged by observation of consumption of dinner on weekdays, and by evaluation of the records made by the participants and kitchen staff during the intervention. For the twelve subjects who were investigated postprandially, the compliance on the 'study day' was judged by observation of consumption.

\section{Statistics}

The fasting variables were analysed by repeated measures ANOVA (GLM Univariate procedure in SPSS). In addition to diet, period was included in the model to test for systematic differences between the three intervention periods. If there was no period effect, it was removed from the model. When the ANOVA showed statistically significant effect of diet, pair-wise comparisons between the three diets were performed with the Bonferonni correction. The postprandial variables were also analysed by repeated measurements ANOVA. Diet, time, and the interaction between time and treatment were included in the model, in addition to the inclusion of the fasting levels as a covariate. The effect of the interaction term and the covariate proved non-significant, and were thus removed from the model. Residuals were examined to check the normality assumption. Pearson's correlation coefficient was estimated to assess associations between PC and FVII. The correlation coefficients were estimated on fasting variables and separately for each diet. A 5\% level of significance was applied in all analyses. The SPSS statistical package version 11.0 (SPSS Inc., Chicago, IL, USA) was used throughout.

\section{Results}

\section{Subjects and dietary compliance}

A total of twenty-five participants completed the study. Five dropped out after the first period and one after the second period. Of the subset of thirteen who were studied postprandially, twelve completed the study. The mean fasting body weights after each of the three dietary periods 
did not differ notably (HSAFA 67.1 (SD 12.0) kg, LSAFA 67.0 (SD 11.9) kg, and HUFA 67.1 (SD 12.0) kg). Evaluation of the dietary compliance showed only minor deviations from the diet.

\section{Test diets}

The results of the duplicate portions and fatty acid composition of the three diets are given in Tables 1 and 2, respectively. The proportions of fat in the three diets were slightly lower than originally planned, $38.4 \%$ energy in the HSAFA diet, $19.7 \%$ energy in the LSAFA diet and $38.2 \%$ energy in the HUFA diet. The energy content was somewhat higher than the calculated $8 \cdot 2 \mathrm{MJ}$ (Table 1).

Table 1. Energy and nutrient content of the three test diets based on duplicate portions*

\begin{tabular}{|c|c|c|c|}
\hline Diet... & HSAFA† & LSAFA† & HUFA \\
\hline Energy (MJ) & $8 \cdot 72$ & $8 \cdot 66$ & $8 \cdot 85$ \\
\hline Protein (\% energy) & 14.9 & $16 \cdot 5$ & $15 \cdot 0$ \\
\hline Fat (\% energy) & 38.4 & $19 \cdot 7$ & $38 \cdot 2$ \\
\hline $\begin{array}{l}\text { Sum of } 12: 0+14: 0+16: 0 \\
\text { (\% energy) }\end{array}$ & $22 \cdot 7$ & $10 \cdot 5$ & $2 \cdot 4$ \\
\hline $\begin{array}{l}\text { Sum of cis monounsaturated } \\
\text { fatty acids (\% energy) }\end{array}$ & $5 \cdot 5$ & 3.5 & $14 \cdot 1$ \\
\hline $\begin{array}{l}\text { Sum of cis polyunsaturated } \\
\text { fatty acids (\% energy) }\end{array}$ & $3 \cdot 9$ & $2 \cdot 3$ & $15 \cdot 6$ \\
\hline Carbohydrates (\% energy) & $46 \cdot 7$ & $63 \cdot 8$ & $46 \cdot 8$ \\
\hline Cholesterol (mg) & $51 \cdot 1$ & $49 \cdot 3$ & $56 \cdot 9$ \\
\hline
\end{tabular}

HSAFA, high-fat diet rich in saturated fatty acids; LSAFA, low-fat diet rich in saturated fatty acids; HUFA, high-fat diet rich in unsaturated fatty acids. *Portions corresponding to an estimated intake of $8.2 \mathrm{MJ} / \mathrm{d}$ were analysed. $\dagger$ Based on the same saturated fatty acid margarine.

Table 2. Fatty acid composition (\% of total fatty acids) of the three test diets ${ }^{*}$

\begin{tabular}{|c|c|c|c|}
\hline \multirow[b]{2}{*}{ Fatty acid } & \multicolumn{3}{|c|}{ Diet } \\
\hline & HSAFA† & LSAFA† & HUFA \\
\hline $6: 0$ & 0.6 & 0.7 & \\
\hline $8: 0$ & 6.4 & $5 \cdot 2$ & 0.2 \\
\hline $10: 0$ & $4 \cdot 7$ & 3.9 & 0.2 \\
\hline $12: 0$ & $34 \cdot 3$ & $27 \cdot 4$ & $2 \cdot 0$ \\
\hline $14: 0$ & $13 \cdot 9$ & $12 \cdot 6$ & 0.7 \\
\hline $16: 0$ & $10 \cdot 8$ & $13 \cdot 4$ & $9 \cdot 4$ \\
\hline $16: 1$ cis & 0.3 & 0.7 & 0.1 \\
\hline $18: 0$ & 3.6 & 4.9 & $8 \cdot 0$ \\
\hline $18: 1$ trans & 0.5 & 0.7 & \\
\hline $18: 1 \mathrm{cis}$ & $14 \cdot 0$ & $17 \cdot 0$ & $36 \cdot 7$ \\
\hline $18: 2$ trans & 0.3 & & \\
\hline $18: 2$ cis & $8 \cdot 6$ & $10 \cdot 2$ & $36 \cdot 2$ \\
\hline $18: 3$ cis & 1.6 & $1 \cdot 7$ & $4 \cdot 6$ \\
\hline $20: 0$ & 0.1 & 0.1 & 0.4 \\
\hline $20: 1$ trans & 0.2 & 0.2 & 0.6 \\
\hline $20: 1$ cis & 0.1 & & \\
\hline $22: 0$ & $<0.1$ & $<0.1$ & 0.2 \\
\hline
\end{tabular}

HSAFA, high-fat diet rich in saturated fatty acids; LSAFA, low-fat diet rich in saturated fatty acids; HUFA, high-fat diet rich in unsaturated fatty acids. * Analysed by GC.

† Based on the same saturated fatty acid margarine.
Effect of experimental diets on fasting levels of activated coagulation factor VII, factor VII protein, factor VII coagulant activity and choline-containing phospholipids

Diet and period effects were both significant for fasting FVIIa levels $(P<0 \cdot 001$ and $P=0 \cdot 01$, respectively $)$. The fasting levels of FVIIa after the HSAFA diet were significantly higher than after both the LSAFA and the HUFA diets $(P<0.01$ and $P=0 \cdot 001$, respectively), whereas no significant difference was found between FVIIa levels after the LSAFA and the HUFA diets (Table 3). The period effect was probably due to two subjects in sequence $\mathrm{ABC}$ with especially high levels of FVIIa after consuming the HSAFA diet. When these two subjects were excluded from the analysis, the period effect was no longer statistically significant, whereas the diet effect was still significant $(P=0 \cdot 001)$. For the other variables, no period effect was found $(P \geq 0 \cdot 13)$. No significant effect of diet was found for fasting levels of FVIIag and FVIIc (Table 3) $(P=0 \cdot 12$, and $P=0 \cdot 12$, respectively). There was a significant overall effect of diet on fasting PC levels $(P<0.001)$. Fasting PC levels after the HSAFA diet were significantly higher than those after the LSAFA and HUFA diets $(P=0.01$ and $P<0.001$, respectively) (Table 3). Moreover, the fasting PC levels after the LSAFA diet were significantly higher than after the HUFA diet $(P<0.001)$.

Effect of experimental diets on postprandial levels of activated coagulation factor VII, factor VII coagulant activity and choline-containing phospholipids

There was a significant effect of both diet and time for FVIIa levels $(P<0.001$ and $P<0.001$, respectively $)$ (Fig. 1 (a)). The HSAFA diet resulted in significantly higher postprandial levels of FVIIa as compared with the HUFA and LSAFA diets $(P=0.05$ and $P<0.001$, respectively). FVIIa levels after the HUFA diet were also significantly higher than after the LSAFA diet $(P=0.03)$. For FVIIc levels, there was no significant effect of diet and time (Fig. 1 (b)). Significant effects of both diet and time in postprandial PC levels were observed $(P<0.001$ and $P<0.001$, respectively) (Fig. 1 (c)). The HSAFA diet resulted in significantly higher postprandial levels of PC as compared with the LSAFA and HUFA diets $(P<0.001$ and $P<0.001$, respectively). Furthermore, the PC levels after the LSAFA diet were significantly higher than after the HUFA diet $(P<0.001)$. No significant interaction was found between time and diet for FVIIa, FVIIc or PC levels.

\section{Associations between fasting levels of choline-containing phospholipids and coagulation factor VII}

No significant associations were found between PC and FVIIa levels after any of the diets (Table 4), whereas significant, positive correlations were found between PC and FVIIag after both the HSAFA $(P=0.03)$ and the HUFA diet $(P=0 \cdot 01)$. Furthermore, significant correlations were found between PC and FVIIc after all diets $(P=0.02$ for both LSAFA and HUFA diets), and correlations of borderline significance after the HSAFA diet $(P=0 \cdot 06)$. 
Table 3. Fasting values at study entry and after consumption of a high-fat diet rich in saturated fatty acids (HSAFA), a low-fat diet rich in saturated fatty acids (LSAFA) and a high-fat diet rich in unsaturated fatty acids (HUFA) for 3 weeks§

(Mean values and standard deviations)

\begin{tabular}{|c|c|c|c|c|c|c|c|c|c|}
\hline & \multirow{2}{*}{$n$} & \multicolumn{2}{|c|}{ Baseline } & \multicolumn{2}{|c|}{ HSAFA } & \multicolumn{2}{|c|}{ LSAFA } & \multicolumn{2}{|c|}{ HUFA } \\
\hline & & Mean & SD & Mean & SD & Mean & SD & Mean & SD \\
\hline FVIIa (mU/ml) & 17 & $61 \cdot 0$ & $22 \cdot 6$ & $66 \cdot 9^{*} \dagger$ & $36 \cdot 3$ & 51.5 & $19 \cdot 1$ & 49.5 & $16 \cdot 8$ \\
\hline FVIIag (ng/ml) & 25 & & & 425 & 83 & 413 & 78 & 406 & 74 \\
\hline FVIIc (\%) & 25 & $115 \cdot 0$ & $26 \cdot 0$ & $124 \cdot 5$ & $25 \cdot 8$ & $117 \cdot 2$ & $26 \cdot 0$ & $115 \cdot 0$ & $25 \cdot 6$ \\
\hline $\mathrm{PC}(\mathrm{mmol} / \mathrm{l})$ & 25 & $2 \cdot 89$ & 0.38 & $3.01^{*} \dagger$ & 0.59 & $2 \cdot 84 \ddagger$ & 0.48 & 2.55 & 0.43 \\
\hline
\end{tabular}

FVIIa, activated coagulation factor VII; FVIlag, FVII protein; FVIIc, FVII coagulant activity; PC, phosphatidylcholine.

* Mean value for the HSAFA diet was significantly different from that for the LSAFA diet $(P \leq 0.01)$.

t Mean value for the HSAFA diet was significantly different from that for the HUFA diet $(P \leq 0.001)$

$\ddagger$ Mean value for the LSAFA diet was significantly different from that for the HUFA diet $(P \leq 0.001)$

$\S$ For details of diets and procedures, see Tables 1 and 2 and p. 330 .

\section{Discussion}

The present controlled dietary crossover study investigated the effects of dietary fat content and fatty acid composition on fasting and postprandial levels of blood FVII and serum $\mathrm{PC}$, and their possible associations.

Our main finding was that consumption of the HSAFA diet for 3 weeks was associated with significantly higher fasting and postprandial levels of FVIIa and PC as compared with consumption of the HUFA and LSAFA diets. These variables were thus dependent both on fatty acid content and on fatty acid composition.

Our results are thus not quite in accordance with previous studies indicating that fasting FVIIa is independent of the dietary fatty acid composition (Larsen et al. 1999; Hunter et al. 2000). The effect of fat quantity on fasting FVIIa, on the other hand, has to our knowledge not been reported previously. Fasting FVIIag levels were unaffected by dietary fat and this is in agreement with several other studies (Marckmann et al. 1990; Brace et al. 1994; Larsen et al. 1999; Hunter et al. 2000). Furthermore, no statistical differences could be demonstrated between the three diets in fasting FVIIc levels, which is in accordance with some other studies reporting that fasting FVIIc is independent of fatty acid composition (Marckmann et al. 1990; Larsen et al. 1999; Hunter et al. 2000). However, in contrast to our results, high-fat diets have been reported to be associated with increased fasting levels of FVIIc compared with low-fat diets (Miller et al. 1986; Marckmann et al. 1993; Brace et al. 1994).

The postprandial FVIIa increase was marked after both the HSAFA and HUFA diets. The highest levels were observed after the HSAFA diet, possibly due to the initially higher fasting levels compared with the HUFA diet. The increase after the LSAFA diet, however, was just discernible. The postprandial FVIIa responses thus differed significantly between the three diets. These results support previous studies reporting an increase in postprandial FVIIa levels after high-fat meals (Kapur et al. 1996; Larsen et al. 1997, 2000; Mennen et al. 1998; Oakley et al. 1998; Sanders et al. 2000). Our findings can also be discussed along with results from previous studies reporting that postprandial FVIIa levels are dependent on fatty acid composition (Larsen et al. 1999; Sanders et al. 2000, 2001; Hunter et al. 2001), although conflicting results exist (Mennen et al. 1998). There was no effect of any diets on postprandial FVIIc levels, which is in agreement with some studies (Larsen et al. 2000; Hunter et al. 2001). However, postprandial FVIIc levels are in other studies reported to increase after high-fat meals (Larsen et al. 1997, 1999; Oakley et al. 1998; Sanders et al. 2000, 2001), and in some studies to decrease after low-fat meals (Larsen et al. 2000; Sanders et al. 2000).

The results from the present and previous studies indicate that dietary fat influences both fasting and postprandial levels of FVII mainly by activation of the inactive zymogen, probably not by altering protein synthesis. FVIIc is a measure of plasma coagulant activity, and includes FVIIa and a certain amount of FVII zymogen activated during the assay (Mennen et al. 1996). Different methods for determination of FVIIc can probably to some extent explain the conflicting results regarding dietary fat content and effect on FVIIc levels, whilst the results on FVIIa are more consistent. It has also been reported that different FVII genotypes are responsible for individual variation in plasma FVII levels (Bernardi et al. 1996), and that the postprandial response to dietary fat is dependent on these genotypes (Mennen et al. 1999; Roche et al. 2000). The frequency of these genotypes differs between populations (Bernardi et al. 1997), and this might also have contributed to the conflicting results. It also should be noted that studies on postprandial effects either investigate the response after several weeks of intervention (chronic effects), or after ingestion of single meals (acute effects). Furthermore, the number of meals used in different studies varies, and mostly oneor two-meal designs have been used. In the present study, however, a four-meal design with blood sampling $1.5 \mathrm{~h}$ after each meal was used. The effects observed in our study have an additive nature as the effect of each meal cannot be differentiated. Our observations are thus more close to real life than studies with a onemeal design. The results from single- or two-meal studies are therefore not directly comparable with the present results.

How dietary fat promotes the activation of FVII is not clear, and this may be different in the fasting and postprandial situations. A relationship to blood lipids such as cholesterol and triacylglycerols has been investigated by 

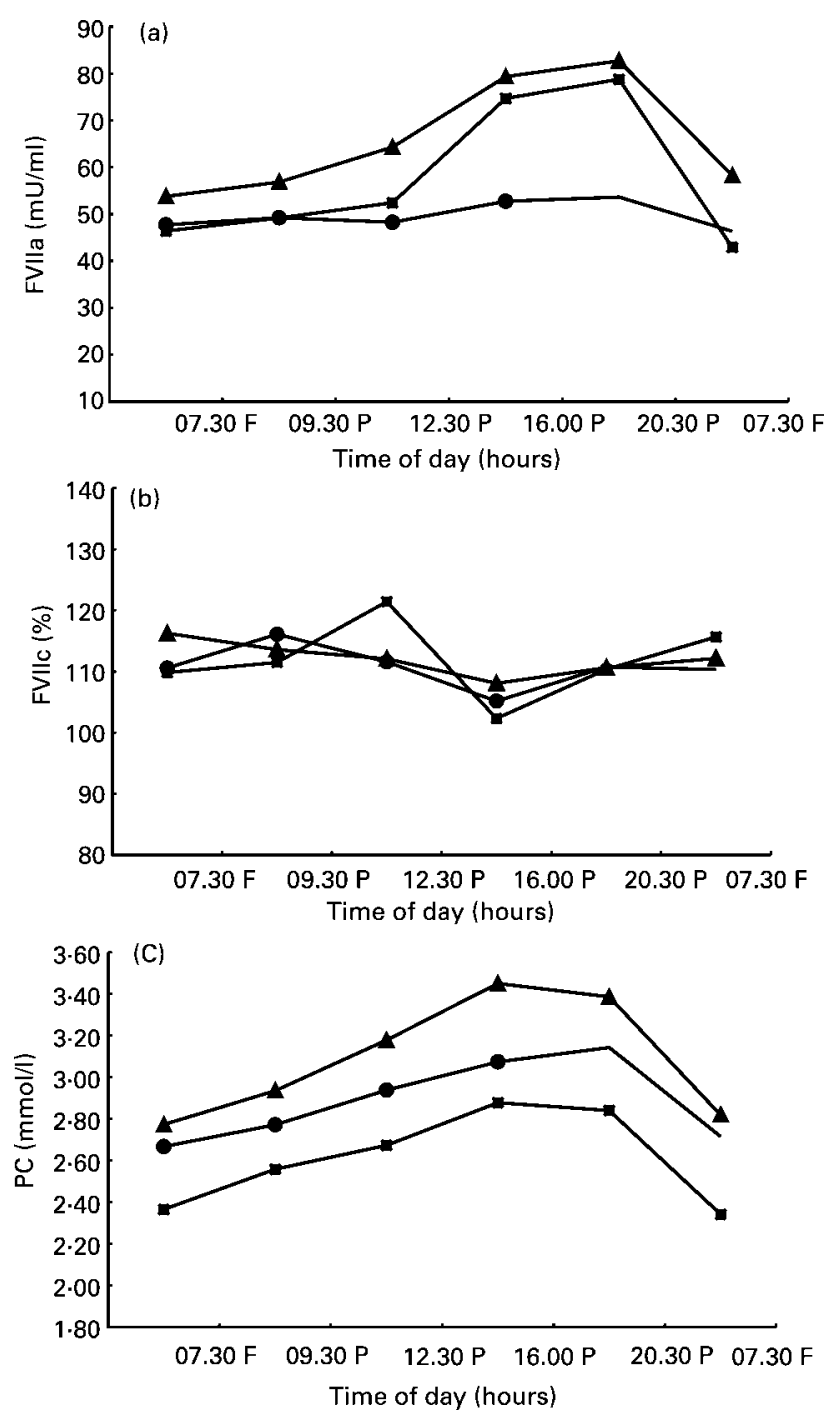

Fig. 1. Mean fasting $(F)$ and postprandial $(P)$ levels of activated coagulation factor VII (FVIla; $n$ 6) (a), factor VII coagulant activity (FVIIc; $n$ 12) (b), and choline-containing phospholipids (PC; $n$ 12) (c) after consumption of a high-fat diet rich in saturated fatty acid (HSAFA; $\mathbf{\Delta}$ ), a low-fat diet rich in saturated fatty acids (LSAFA; $\bullet$ ), and a high-fat diet rich in unsaturated fatty acids (HUFA; $\mathbf{0}$ ) for 3 weeks. Postprandial samples were collected $1.5 \mathrm{~h}$ after each meal. Meals were served at 08.00 hours (breakfast), 11.00 hours (lunch), 14.30 hours (dinner) and 19.00 hours (evening meal). Differences between diets were analysed as described on p. 000 . For FVlla, significant differences were found between diets HSAFA and LSAFA $(P<0.001)$, HSAFA and HUFA $(P=0.05)$, and between LSAFA and HUFA $(P=0.03)$. For PC, significant differences were found between diets HSAFA and LSAFA $(P<0.001)$, HSAFA and HUFA $(P<0.001)$, and between LSAFA and HUFA $(P<0.001)$.

several authors (Marckmann et al. 1990, 1992, 1993; Kapur et al. 1996; Larsen et al. 2000; Sanders et al. 2000, 2001), but no conclusive results have been presented. A link between FVII and PL was earlier suggested by Dalaker et al. (1985). In a recent study serum PC were the main determinant of FVIIc and FVIIa in the most common FVII genotypes of the R353Q and the $5^{\prime} \mathrm{F} 7$ polymorphisms on the FVII gene (Mariani et al. 1999). This indicated that plasma lipids contributed to FVII levels through the PL content, and that the degree of this contribution was strictly dependent on FVII genotypes.

In the present study the fasting and postprandial PC response was mainly dependent on fatty acid composition and to a lesser extent on fat quantity. The highest fasting and postprandial PC levels were seen after the HSAFA diet, followed by the LSAFA diet, whereas the HUFA diet resulted in the lowest PC levels. The postprandial increase was marked after all diets, but initially high levels of fasting PC resulted in higher postprandial levels. Serum PL have been reported to increase postprandially after ingestion of fat-rich meals, due to increased PL content in the chylomicrons, VLDL, and HDL fractions (Havel, 1957). Others have also reported high levels of HDL PL after fat-rich meals (Havel et al. 1973; Groener et al. 1998). In the present study, however, the different contributions from the lipoprotein fractions to PC levels cannot be distinguished.

Fasting PC levels correlated significantly with both FVIIc and FVIIag levels, whereas the correlation coefficients for FVIIa were insignificant, probably due to the small sample size (Table 4). These results are supported by the report from Mariani et al. (1999) where the associations between FVII and PC were most evident in fasting subjects. Furthermore, the association between FVII and PL found by Dalaker et al. (1985) were also found in fasting subjects.

Our results, however, do not support the assumption that the postprandial increase in FVIIa is linked to PC. The postprandial levels of both FVIIa and PC were highest after the HSAFA diet. However, the postprandial FVIIa levels were lowest after the LSAFA diet, whereas the lowest PC concentration was found after consumption of the HUFA diet, thus indicating that postprandial levels of FVIIa and PC are differently influenced by dietary fat.

The limited number of individuals investigated for FVIIa, analytical imprecision at high values, and the statistical period effect obtained in the fasting study have to be taken into account in the interpretation of the results. Furthermore, for methodological reasons our investigation has been limited to the determination of PC, which is a highly reproducible and standardized method. PC average about $90 \%$ of the total PL in plasma (Mariani et al. 1999), and it cannot be excluded that other PL compounds could play a role in an interaction with FVII.

In conclusion, our results showed that dietary fat content and fatty acid composition influence both fasting and postprandial levels of FVIIa and PC. In addition, strong associations between the fasting levels of FVII and PC were present. However, the results do not support the assumption that serum $\mathrm{PC}$ is linked to postprandial FVII activation.

\section{Acknowledgements}

The study was made possible by grants from the Norwegian Research Council, the food company Mills DA and G. Aakre's Student Fund. Thanks are given to all participants for their cooperation, and to University College of Akershus for providing kitchen facilities. Also 
Table 4. Pearson's correlation coefficients $(r)$ for associations between fasting levels of choline-containing phospholipids (PC) and coagulation factor VII (FVII) after the three diets*

\begin{tabular}{|c|c|c|c|c|c|c|c|}
\hline \multirow[b]{2}{*}{ Diet... } & \multirow[b]{2}{*}{$n$} & \multicolumn{2}{|c|}{ HSAFA } & \multicolumn{2}{|c|}{ LSAFA } & \multicolumn{2}{|c|}{ HUFA } \\
\hline & & $r$ & $P$ value & $r$ & $P$ value & $r$ & $P$ value \\
\hline PC v. FVIIa & 17 & 0.36 & 0.16 & 0.41 & 0.11 & 0.29 & 0.26 \\
\hline PC v. FVIlag & 25 & 0.44 & 0.03 & 0.27 & 0.19 & 0.49 & 0.01 \\
\hline PC v. FVIIc & 25 & 0.39 & 0.06 & 0.48 & 0.02 & 0.47 & 0.02 \\
\hline
\end{tabular}

acknowledged are Anne Lise Brantsæter, Anita Blomfeldt, Connie Weng, Anita Kavlie, Bente Rønning and Frank Brosstad for assisting in practical and technical aspects of the study.

\section{References}

Bernardi F, Arcieri P, Bertina RM, et al. (1997) Contribution of factor VII genotype to activated FVII levels. Differences in genotype frequencies between northern and southern European populations. Arterioscler Thromb Vasc Biol 17, 2548-2553.

Bernardi F, Marchetti G, Pinotti M, et al. (1996) Factor VII gene polymorphisms contribute about one third of the factor VII level variation in plasma. Arterioscler Thromb Vasc Biol 16, $72-76$.

Brace LD, Gittler-Buffa C, Miller GJ, et al. (1994) Factor VII coagulant activity and cholesterol changes in premenopausal women consuming a long-term cholesterol-lowering diet. Arterioscler Thromb 14, 1284-1289.

Cooper JA, Miller GJ, Bauer KA, et al. (2000) Comparison of novel hemostatic factors and conventional risk factors for prediction of coronary heart disease. Circulation 102, $2816-2822$.

Dalaker K, Hjermann I \& Prydz H (1985) A novel form of factor VII in plasma from men at risk for cardiovascular disease. $\mathrm{Br} \mathrm{J}$ Haematol 61, 315-322.

Eriksson-Berg M, Silveira A, Orth-Gomer K, Hamsten A \& Schenck-Gustafsson K (2001) Coagulation factor VII in middle-aged women with and without coronary heart disease. Thromb Haemost 85, 787-792.

Folsom AR, Wu KK, Shahar E \& Davis CE (1993) Association of hemostatic variables with prevalent cardiovascular disease and asymptomatic carotid artery atherosclerosis. The Atherosclerosis Risk in Communities (ARIC) Study Investigators. Arterioscler Thromb 13, 1829-1836.

Groener JE, Scheek LM, van Ramshorst E, Krauss XH \& van Tol A (1998) Delayed increase in high density lipoprotein-phospholipids after ingestion of a fat load in normolipidemic patients with coronary artery disease. Atherosclerosis 137, 311-319.

Havel RJ (1957) Early effects of fat ingestion on lipids and lipoproteins of serum in man. J Clin Invest 36, 848-854.

Havel RJ, Kane JP \& Kashyap ML (1973) Interchange of apolipoproteins between chylomicrons and high density lipoproteins during alimentary lipemia in man. J Clin Invest 52, 32-38.

Heinrich J, Balleisen L, Schulte H, Assmann G \& van de Loo J (1994) Fibrinogen and factor VII in the prediction of coronary risk. Results from the PROCAM study in healthy men. Arterioscler Thromb 14, 54-59.
Hunter KA, Crosbie LC, Horgan GW, Miller GJ \& Dutta-Roy AK (2001) Effect of diets rich in oleic acid, stearic acid and linoleic acid on postprandial haemostatic factors in young healthy men. Br J Nutr 86, 207-215.

Hunter KA, Crosbie LC, Weir A, Miller GJ \& Dutta-Roy AK (2000) A residential study comparing the effects of diets rich in stearic acid, oleic acid, and linoleic acid on fasting blood lipids, hemostatic variables and platelets in young healthy men. J Nutr Bioch 11, 408-416.

Junker R, Heinrich J, Schulte H, van de Loo J \& Assmann G (1997) Coagulation factor VII and the risk of coronary heart disease in healthy men. Arterioscler Thromb Vasc Biol 17, $1539-1544$.

Kapur R, Hoffman CJ, Bhushan V \& Haltin MB (1996) Postprandial elevation of activated factor VII in young adults. Arterioscler Thromb Vasc Biol 16, 1327-1332.

Larsen LF, Bladbjerg EM, Jespersen J \& Marckmann P (1997) Effects of dietary fat quality and quantity on postprandial activation of blood coagulation factor VII. Arterioscler Thromb Vasc Biol 17, 2904-2909.

Larsen LF, Jespersen J \& Marckmann P (1999) Are olive oil diets antithrombotic? Diets enriched with olive, rapeseed, or sunflower oil affect postprandial factor VII differently. Am J Clin Nutr 70, 976-982.

Larsen LF, Marckmann P, Bladbjerg EM, Ostergaard PB, Sidelmann J \& Jespersen J (2000) The link between high-fat meals and postprandial activation of blood coagulation factor VII possibly involves kallikrein. Scand J Clin Lab Invest 60 , $45-54$.

Marckmann P, Sandstrom B \& Jespersen J (1990) Effects of total fat content and fatty acid composition in diet on factor VII coagulant activity and blood lipids. Atherosclerosis 80, 227-233.

Marckmann P, Sandstrom B \& Jespersen J (1992) Fasting blood coagulation and fibrinolysis of young adults unchanged by reduction in dietary fat content. Arterioscler Thromb 12, 201-205.

Marckmann P, Sandstrom B \& Jespersen J (1993) Favorable long-term effect of a low-fat/high-fiber diet on human blood coagulation and fibrinolysis. Arterioscler Thromb 13, 505-511.

Mariani G, Bernardi F, Bertina R, et al. (1999) Serum phospholipids are the main environmental determinants of activated factor VII in the most common FVII genotype. European Union Concerted Action "Clotart". Haematologica 84, 620-626.

Meade TW, Mellows S, Brozovic M, et al. (1986) Haemostatic function and ischaemic heart disease: principal results of the Northwick Park Heart Study. Lancet ii, 533-537.

Mennen L, de Maat M, Meijer G, et al. (1998) Factor VIIa response to a fat-rich meal does not depend on fatty acid composition: a randomized controlled trial. Arterioscler Thromb Vasc Biol 18, 599-603. 
Mennen LI, de Maat MP, Meijer G, et al. (1999) Postprandial response of activated factor VII in elderly women depends on the R353Q polymorphism. Am J Clin Nutr 70, 435-438.

Mennen LI, Schouten EG, Grobbee DE \& Kluft C (1996) Coagulation factor VII, dietary fat and blood lipids: a review. Thromb Haemost 76, 492-499.

Merlini PA, Ardissino D, Oltrona L, Broccolino M, Coppola R \& Mannucci PM (1995) Heightened thrombin formation but normal plasma levels of activated factor VII in patients with acute coronary syndromes. Arterioscler Thromb Vasc Biol 15, $1675-1679$.

Miller GJ, Bauer KA, Barzegar S, Cooper JA \& Rosenberg RD (1996) Increased activation of the haemostatic system in men at high risk of fatal coronary heart disease. Thromb Haemost 75, 767-771.

Miller GJ, Martin JC, Webster J, et al. (1986) Association between dietary fat intake and plasma factor VII coagulant activity - a predictor of cardiovascular mortality. Atherosclerosis 60, 269-277.

Moor E, Silveira A, van't Hooft F, et al. (1995) Coagulation factor VII mass and activity in young men with myocardial infarction at a young age. Role of plasma lipoproteins and factor VII genotype. Arterioscler Thromb Vasc Biol 15, 655-664.

Morrissey JH, Macik BG, Neuenschwander PF \& Comp PC (1993) Quantitation of activated factor VII levels in plasma using a tissue factor mutant selectively deficient in promoting factor VII activation. Blood 81, 734-744.

Muller H, Lindman AS, Brantsæter A \& Pedersen JI (2003) The serum LDL/HDL cholesterol ratio is influenced more favorably by exchanging saturated with unsaturated fat than by reducing saturated fat in the diet of women. $J$ Nutr 133, 78-83.

Muller H, Seljeflot I, Solvoll K \& Pedersen JI (2001) Partially hydrogenated soybean oil reduces postprandial t-PA activity compared with palm oil. Atherosclerosis 155, 467-476.

Oakley FR, Sanders TA \& Miller GJ (1998) Postprandial effects of an oleic acid-rich oil compared with butter on clotting factor VII and fibrinolysis in healthy men. Am J Clin Nutr 68, $1202-1207$.

Roche HM, Black IL, Noone E, Tully AM, Whitehead AS \& Gibney MJ (2000) Postprandial factor VII metabolism: the effect of the R353Q and 10 bp polymorphisms. Br J Nutr 83, 467-472.

Sanders TA, de Grassi T, Miller GJ \& Morrissey JH (2000) Influence of fatty acid chain length and cis/trans isomerization on postprandial lipemia and factor VII in healthy subjects (postprandial lipids and factor VII). Atherosclerosis 149, 413-420.

Sanders TA, Oakley FR, Cooper JA \& Miller GJ (2001) Influence of a stearic acid-rich structured triacylglycerol on postprandial lipemia, factor VII concentrations, and fibrinolytic activity in healthy subjects. Am J Clin Nutr 73, 715-721.

Takayama M, Itoh S, Nagasaki T \& Tanimizu I (1977) A new enzymatic method for determination of serum cholinecontaining phospholipids. Clin Chim Acta 79, 93-98.

World Health Organization (1985) Energy and Protein Requirements. Report of a Joint FAO/WHO/UNU Expert Consultation. Technical Report Series no. 724. Geneva, Switzerland: WHO. 